\title{
Histomorphometric evaluation of periodontal compression and tension sides during orthodontic tooth movement in rats
}

\author{
Rodrigo Castellazzi Sella', Marcos Rogério de Mendonça², Osmar Aparecido Cuoghi², Tien Li An ${ }^{3}$
}

Objective: The purpose of this study was to evaluate the thickness of the periodontal ligament of rat molars during orthodontic tooth movement (OTM).

Methods: Thirty Wistar rats were divided into three groups of 10 animals each: GI, GII and GIII and the mice were euthanized at 7, 14 and 21 days, respectively. Experimental subjects were compared to their respective controls by the Mann-Whitney test. Comparison of values between compression and tension sides were performed during the same and different time periods through Analysis of Variance (ANOVA), Kruskal-Wallis test and, subsequently, Tukey's test.

Results: Groups GI and GII showed decreased PDL size in the apical regions of the mesiobuccal root and in the cervical region of the distobuccal root. There was also an increased PDL in the cervical regions of the mesiobuccal root, apical region of the distobuccal root and middle region of both roots.

Conclusion: The reduction and increase in PDL size were seen in the same root, which characterizes tooth inclination. The apical, middle and cervical regions were compared with one another in each time period and at three times: 7, 14 and 21 days. They were also compared in each region, confirming a tipping movement in GI and GII and a gradual decreased intensity between GI to GII, reaching normal dimension in GIII.

Keywords: Tooth movement. Periodontal ligament. Periodontium.

${ }^{1}$ Specialist in Orthodontics and Facial Orthopedics, UEL. MSc and PhD in Dentistry, concentration in Orthodontics, FOA-UNESP. Professor of Department of Anatomy of Center of Biological Sciences, disciplines of Human Anatomy and Dental Anatomy, UEL. Professor and Coordinator of Specialization in Orthodontics, UNICSUL.

\footnotetext{
${ }^{2} \mathrm{PhD}$ and Associate Professor of Department of Child and Social Dentistry, discipline of Preventive Orthodontics, FOA-UNESP.

${ }^{3}$ Professor of Orthodontics, FOA-UNESP and School of Health Sciences, Brasilia University.
}

How to cite this article: Sella RC, Mendonça MR, Cuoghi OA, An TL. Histomorphometric evaluation of periodontal compression and tension sides during orthodontic tooth movement in rats. Dental Press J Orthod. 2012 May-June;17(3):108-17.

Submitted: May 19, 2009 - Revised and accepted: April 12, 2010

» The authors report no commercial, proprietary, or financial interest in the products or companies described in this article..

Contact address: Rodrigo Castellazzi Sella

R. Caracas, 555 - Zip code: 86050-070 - Londrina/PR - Brazil

E-mail: rodrigosella@hotmail.com 


\section{INTRODUCTION}

Histology is one of the areas of biology that has enabled numerous advances in Orthodontics, since it is a science that studies the microscopic structures of tissues and organs and is, therefore, intimately related to the study of tooth movement. Pioneer studies of Sandstedt, ${ }^{24}$ Oppenheim, ${ }^{16}$ Reitna, ${ }^{19}$ Roberts,${ }^{21,22}$ Rugh $^{23}$ and Davidovitch ${ }^{8}$ are classical and constitute the basis of orthodontic knowledge in this area.

The periodontal ligament (PDL), located between bone and tooth, is the physiological mediator of orthodontic treatment. ${ }^{8}$ This is a modified periosteum ${ }^{4}$ that is capable of causing tissue resorption and bone deposition. ${ }^{8}$ PDL cellular kinetics has provided information that defined the events of proliferation and differentiation of orthodontic reaction, essential for the mechanical induction of osteogenesis and osteoclasia. $^{15,17,25,28,29}$

Tooth displacement occurs in response to an induced force and comprises three elements: Initial stress, plateau and progressive tooth movement. ${ }^{21}$ In the first week, stress occurs through dental displacement in the PDL, bone resistance and extrusion. ${ }^{22}$ During this time, initial PDL stress varies according to PDL thickness, root length and periodontal health. ${ }^{21}$ Initial tooth displacement happens in seconds, but effective PDL compression requires one to three hours. ${ }^{22}$ One minute after application of continuous force to a murine first molar, there are changes in electrical potential of the periodontium, which in turn generate PDL osteogenic and osteoclastic responses $^{22}$, in other words, force application triggers a cascade of cellular events in the PDL. ${ }^{8}$ Bone tissue is removed by osteoclasts and new bone is deposited by osteoblasts as periodontal structures adapt, keeping the teeth in their new environment. ${ }^{15,17,25,28,29}$

Although histological changes in the periodontium associated with orthodontic induction of osteoclasia and osteogenesis ${ }^{15,17,25,28,29}$ and the phenomenon of bone bending ${ }^{22}$ are of common knowledge in the literature, little information is available concerning the histometric behavior of PDL compression and tension sides during tooth movement in different root regions and at different time periods. Such gap spurred authors to conduct this study, which aimed to assess the PDL thickness first molars of rats undergoing orthodontic tooth movement (OTM).

\section{MATERIAL AND METHODS \\ Sample selection and distribution}

Thirty 2.5 to 3-month-old male Wistar rats (Rattus norvegicus, albino), weighing between 250 and $350 \mathrm{~g}$, were used in this experiment. The animals were provided by the Animal House of FOA-UNESP and were fed with ground feed (Produtor Activated Feed, Anderson \& Clayton S.A., Laboratório Abbott do Brasil, São Paulo, Brazil) and water ad libitum .

The experimental models were divided into three groups, composed of 10 animals each:

» Group I (GI): 7 days of OTM.

» Group II (GII): 14 days of OTM.

" Group III (GIII): 21 days of OTM.

In all 3 groups, the right upper first molars were subjected to OTM and the left upper first molars were used as controls.

\section{Placement and activation of orthodontic devices}

Before the experimental procedures, the animals were kept in cages for 7 days in a 12/12-hour cycle with constant temperature.

To place the mechanical device, the muscle relaxant Xylazine hydrochloride was used (Dopaser, Calier Laboratorios SA, Spain) at a ratio of $0.03 \mathrm{ml} / 100 \mathrm{~g}$ body weight with the anesthetic ketamine hydrochloride (Vetaset, Fort Dodge Animal Health, Fort Dodge, Iowa, USA) at a ratio of $0.07 \mathrm{ml} / 100 \mathrm{~g}$ body weight. Both drugs were applied by intramuscular injection.

This research employed an orthodontic device designed by Heller and Nanda ${ }^{9}$ and modified by Consolaro and Martins-Ortiz ${ }^{5}$ (Fig 1).

The device consisted of a $4 \mathrm{~mm}$ length stainless steel coil-spring (0.006 x 0.022 HI-TIITM, 3M Unitek, USA). ${ }^{5}$ In order to increase retention, 0.20 $\mathrm{mm}$ ligature wires (Morelli, Sorocaba, Brazil) were adapted to the molars and incisors and covered with chemically cured composite resin (Concise 3M Unitek, Sumaré, Brazil). The amount of activation was measured by means of a caliper until it was expanded to $6 \mathrm{~mm},{ }^{5}$ which is equivalent to $40 \mathrm{cN}^{12}$ or $40 \mathrm{~g}$ of applied force. Force magnitude was set in advance by means of a 28-450 g tension gauge (Dentaurum, Germany). Due to continuous eruption of the rat incisors, the position of the ligature wires was evaluated on a weekly basis. There was no need for readjusting. 


\section{Euthanasia and specimen preparation}

After 7 (GI), 14 (GII) and 21 (GIII) days, the animals were euthanized by overdose of anesthetic and then decapitated. ${ }^{25}$ The right maxillary quadrant was used in the experimental group while the left maxillary quadrant was used as control.

Parafin-embedded samples were cut into serial sections of $6 \mu \mathrm{m}$ thickness, showing the mesiobuccal and distobuccal roots. ${ }^{9}$ The sections were made in the mesiodistal direction in the first upper molars, parallel to the long axis of the teeth for microscopic analysis in the furca region. ${ }^{5}$ Subsequently the material was stained with hematoxylin and eosin.

\section{Scanning of histological sections}

For histometric analysis, the most central section of each tooth was selected and captured using a digital camera (AxioCam MRc5, Carl Zeiss MicroImaging, Gmbh, Germany) coupled to an optical microscope (Leitz Gmbh Aristoplan, Germany) in a $4 \mathrm{X}$ objective using a software (Axio Vision 4.5, Carl Zeiss, Germany) installed on a computer.

\section{Tracing}

After scanning of the histological sections, tracing was performed on the roots to measure the PDL size in different regions (Fig 2).

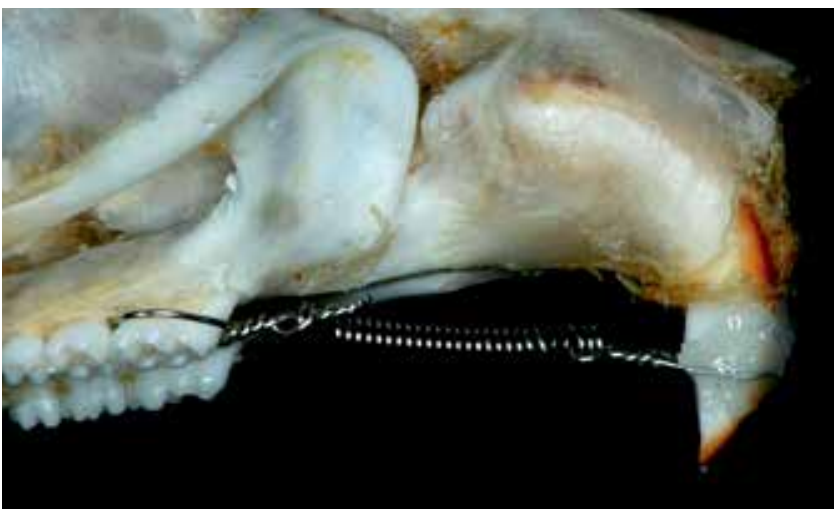

Figure 1- Appliance inducing tooth movement.
The procedure was adapted from the method proposed by King: ${ }^{13}$

\section{Line 1}

Long axes of mesiobuccal and distobuccal roots in the image of the root canal.

\section{Line 2}

Perpendicular to the long axis of the root, in the most apical point of the tooth root, bounded by the tooth and bone surfaces A modification of the method proposed by King, who advocated the use of the most apical point of the PDL.

\section{Line 3}

Perpendicular to the long axis of the root in the most cervical point of the inter-radicular alveolar bone crest, bounded by the tooth and bone surfaces.

\section{Line 4}

Perpendicular to the long axis of the root in the midpoint between lines 2 and 3, bounded by the tooth and bone surfaces.

Extensions of Lines 2, 3 and 4 over the PDL were histometrically analyzed by the ImageLab 2000 software (DiracomBio Informática Ltda., Vargem Grande

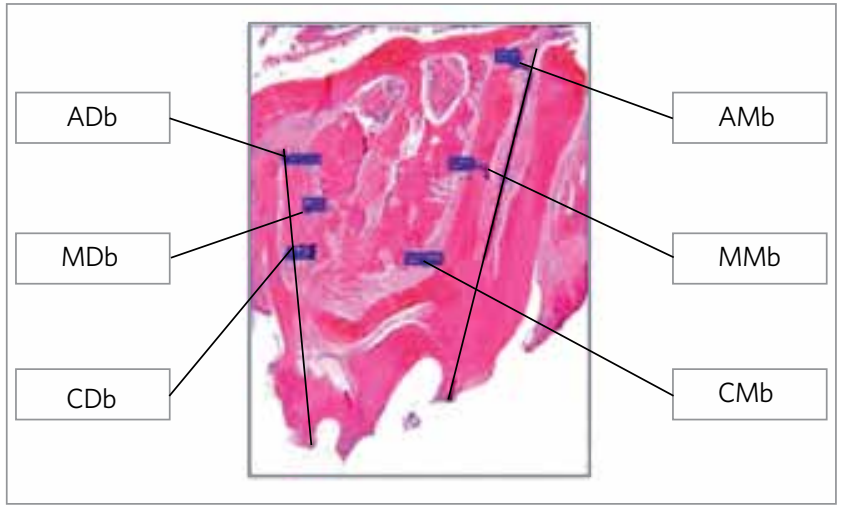

Figure 2 - Tracing of evaluated periodontal ligament regions: Apical distobuc$\mathrm{cal}(\mathrm{ADb})$ middle distobuccal (MDb) and cervical distobuccal (CDb), apical mesiobuccal (AMb), middle mesiobuccal (MMb) and cervical mesiobuccal $(\mathrm{CMb})$. 
do Sul, Brazil), determining PL thickness in metric units at the apical, middle and cervical levels.

\section{Measures evaluated}

Linear measure terminology indicates the root in question, mesiobuccal (M) or distobuccal (D); the region evaluated, apical (Ap), middle (Md) or cervical (Ce); the time period in which the murine molar was submitted to OTM, 7 days (GI), 14 days (GII) and 21 days (GIII); and, additionally, the condition of the tooth being assessed, moved (m) or control (c) tooth.

\section{STATISTICAL ANALYSIS}

SigmaStat software (Advisory Statics for Scientists, version 3.1, SPSS, Chicago, USA) was utilized. The mean values of experimental groups GI, GII and GIII were compared with their respective controls by the Mann-Whitney test $(\mathrm{p}<0.05)$. Comparisons between regions in the same period of time and in different time periods in the same region were performed by analysis of variance (ANOVA, Kruskal-Wallis $-\mathrm{p}<0.05$ ). When ANOVA detected a statistical difference, multiple comparisons were determined by Tukey's test.

\section{METHOD ERROR}

Method error was obtained by randomly selecting one of the roots in two groups. Measurements were performed twice by the same operator and at different periods. ${ }^{10}$ This repetition revealed the random error by the Dahlberg formula: $\mathrm{S}_{\mathrm{e}}{ }^{2}=\Sigma \mathrm{d}^{2} / 2 \mathrm{n}$, where $\mathrm{S}_{\mathrm{e}}$ represents Dahlberg's error, ${ }^{7} \Sigma \mathrm{d}^{2}$ is the sum of squares of differences between the first and second measurements and $2 \mathrm{n}$ represents twice the number of cases that the measurements were repeated. To evaluate systematic error (bias), Mann-Whitney test was employed. ${ }^{10}$

Table 1 - Mean differences, t values (bias), probability and significance levels $(p)$ and Dahlberg's random error obtained in Gl.

\begin{tabular}{ccccc} 
Region / Time & $\begin{array}{c}\text { Difference } \\
\text { mean }\end{array}$ & $\mathbf{t}$ & $\mathbf{p}$ & Dahlberg \\
\hline ADb7m & 0.001 & 0.277 & 0.785 & 0.003872983 \\
MDb7m & 0.002 & 0.577 & 0.571 & 0.003162278 \\
CDb7m & -0.003 & -1.152 & 0.264 & 0.003872983 \\
ADb7c & 0.003 & 0.878 & 0.391 & 0.003872983 \\
MDb7c & 0.001 & 0.342 & 0.736 & 0.003872983 \\
CDb7c & 0.002 & 0.647 & 0.526 & 0.003162278 \\
\hline
\end{tabular}

\section{RESULTS}

Tables 1 and 2 show the method error. Probability and significance levels $(\mathrm{P})$ correspond to the systematic error (bias), ${ }^{10}$ while the values obtained through Dahlberg's formula ${ }^{7}$ determine the random error. The method showed no systematic or random errors and provided results within acceptable parameters without compromising the reliability of the findings of this research.

Tables 3, 4 and 5 refer to GI, GII and GIII, respectively, and show a comparison between the experimental and control groups in each region (apical, middle or cervical) of the mesiobuccal or distobuccal roots.

There were statistically significant differences $(p<0.05)$ in the three regions of both roots in GI and GII (Tables 3 and 4), when comparisons were made between teeth that were either moved or not moved.

However, comparisons in GIII exhibited no statistically significant differences $(\mathrm{p}<0.05)$ between moved and not-moved teeth in the three regions of both roots (Tables 3 and 4).

Tables 6 and 7 show a comparison between apical, middle and cervical measurements in the mesiobuccal and distobuccal roots, respectively in GI, GII or GIII.

The results showed significant differences between the apical region of the mesiobuccal root and the middle and cervical regions $(\mathrm{p}<0.05)$ in GI and GII, which exhibited similar values (Table 6). A comparison between the apical, middle and cervical regions in GIII showed no statistically significant difference.

The data also revealed statistical differences between the cervical region of the mesiobuccal root and the middle and cervical regions $(\mathrm{p}<0.05)$ in GI and GII, which exhibited statistically similar values (Table 6). A comparison between apical, middle and cervical regions in GIII showed no statistically significant difference.

Table 2 - Mean differences, $t$ values (bias), probability and significance levels ( $p$ ) and Dahlberg's random error obtained in GIII.

\begin{tabular}{ccccc}
\hline Region / Time & $\begin{array}{c}\text { Difference } \\
\text { mean }\end{array}$ & $\mathbf{t}$ & $\mathbf{p}$ & Dahlberg \\
\hline AMb21m & 0.000 & 0.000 & 1.000 & 0.004472136 \\
MMb21m & -0.001 & -0.361 & 0.722 & 0.003872983 \\
\hline CMb21m & -0.0002 & -0.0974 & 0.923 & 0.003193744 \\
AMb21c & 0.001 & 0.372 & 0.714 & 0.003872983 \\
\hline MMb21c & 0.003 & 1.152 & 0.264 & 0.003872983 \\
CMb21c & -0.001 & -0.277 & 0.785 & 0.003872983 \\
\hline
\end{tabular}


Table 3 - Means and standard deviations of different thicknesses of periodontal ligament of mesiobuccal (Mb) and distobuccal (Db) roots, moved teeth $(\mathrm{m})$ and controls (c) in GI (7 days) in apical (A) middle (M) and cervical (C) regions, and probability and significance levels ( $\mathrm{p}$ )

\begin{tabular}{cccccccc} 
& \multicolumn{3}{c}{ Periodontal ligament mesiobuccal root } & & & \multicolumn{2}{c}{ Periodontal ligament distobuccal root } \\
Region / Time & Mean & Standard Deviation & $\mathbf{p}$ & Region / Time & Mean & Standard Deviation \\
\hline AMb7m & 0.109 (A) & 0.00316 & $<0.001^{\star}$ & ADb7m & $0.148(A)$ & 0.00789 \\
AMb7c & 0.127 (B) & 0.00675 & & ADb7c & $0.127(B)$ & 0.00675 \\
MMb7m & 0.148 (A) & 0.00789 & $<0.001^{\star}$ & MDb7m & $0.146(A)$ & 0.00699 & $<0.001^{\star}$ \\
MMb7c & 0.128 (B) & 0.00632 & & MDb7c & $0.128(B)$ & 0.00632 \\
CMb7m & 0.149 (A) & 0.00994 & $<0.001^{\star}$ & CDb7m & 0.107 (A) & 0.00483 & $<0.001^{\star}$ \\
CMb7c & 0.128 (B) & 0.00632 & & CDb7c & 0.127 (B) & 0.00675 \\
\hline
\end{tabular}

${ }^{*}$ Different letters: Statistically significant differences indicated by the Mann-Whitney test $(p<0.05)$.

Table 4 - Means and standard deviations of different thicknesses of periodontal ligament of mesiobuccal (Mb) and distobuccal (Db) roots, moved teeth $(\mathrm{m})$ and controls $(\mathrm{c})$ in $\mathrm{GI}(14$ days) in apical $(\mathrm{A})$ middle $(\mathrm{M})$ and cervical $(\mathrm{C})$ regions, and probability and significance levels $(\mathrm{p})$.

\begin{tabular}{|c|c|c|c|c|c|c|c|}
\hline \multicolumn{4}{|c|}{ Periodontal ligament mesiobuccal root } & \multicolumn{4}{|c|}{ Periodontal ligament distobuccal root } \\
\hline Region / Time & Mean & Standard Deviation & $\mathbf{p}$ & Region / Time & Mean & Standard Deviation & $\mathbf{p}$ \\
\hline AMb14m & $0.117(A)$ & 0.00483 & $<0.001^{\star}$ & ADb14m & $0.139(A)$ & 0.00876 & $0.005^{\star}$ \\
\hline AMb14c & $0.128(B)$ & 0.00632 & & ADb14c & $0.128(B)$ & 0.00632 & \\
\hline MMb14m & $0.139(\mathrm{~A})$ & 0.00876 & $0.005^{\star}$ & MDb14m & $0.138(A)$ & 0.00789 & $0.004^{*}$ \\
\hline MMb14c & $0.128(B)$ & 0.00632 & & MDb14c & $0.127(B)$ & 0.00675 & \\
\hline CMb14m & $0.140(A)$ & 0.00816 & $0.003^{\star}$ & CDb14m & $0.116(A)$ & 0.00516 & $<0.001^{\star}$ \\
\hline CMb14c & 0.129 (B) & 0.00568 & & CDb14c & $0.128(B)$ & 0.00632 & \\
\hline
\end{tabular}

${ }^{*}$ Different letters: Statistically significant differences indicated by the Mann-Whitney test $(p<0.05)$.

Table 5 - Means and standard deviations of different thicknesses of periodontal ligament of mesiobuccal (Mb) and distobuccal (Db) roots, moved teeth $(\mathrm{m})$ and controls (c) in $\mathrm{Gl}$ (21 days) in apical (A) middle (M) and cervical (C) regions, and probability and significance levels ( $p$ ).

\begin{tabular}{|c|c|c|c|c|c|c|c|}
\hline \multicolumn{4}{|c|}{ Periodontal ligament mesiobuccal root } & \multicolumn{4}{|c|}{ Periodontal ligament distobuccal root } \\
\hline Region / Time & Mean & Standard Deviation & p & Region / Time & Mean & Standard Deviation & p \\
\hline $\mathrm{AMb} 21 \mathrm{~m}$ & $0.127(A)$ & 0.00675 & 1.000 & ADb21m & $0.130(A)$ & 0.00667 & 0.500 \\
\hline AMb21c & $0.127(A)$ & 0.00675 & & ADb21c & $0.128(A)$ & 0.00632 & \\
\hline MMb21m & $0.130(A)$ & 0.00667 & 0.500 & $\mathrm{MDb} 21 \mathrm{~m}$ & $0.129(A)$ & 0.00568 & 0.714 \\
\hline MMb21c & $0.128(A)$ & 0.00632 & & MDb21c & $0.128(A)$ & 0.00632 & \\
\hline $\mathrm{CMb} 21 \mathrm{~m}$ & $0.131(A)$ & 0.00568 & 0.169 & $\mathrm{CDb} 21 \mathrm{~m}$ & $0.124(A)$ & 0.00516 & 0.054 \\
\hline CMb21c & $0.127(A)$ & 0.00675 & & $\mathrm{CDb} 21 \mathrm{c}$ & $0.129(A)$ & 0.00568 & \\
\hline
\end{tabular}

*Different letters: Statistically significant differences indicated by the Mann-Whitney test $(p<0.05)$.

Tables 8 and 9 show a comparison between apical, middle and cervical regions of the mesiobuccal and distobuccal roots, respectively in GI, GII and GIII. The only statistically significant difference $(\mathrm{p}<0.05)$ was found between periods of 7 and 21 days in the apical, middle and cervical regions of the mesiobuccal and distobuccal roots.

\section{DISCUSSION}

Teeth can move physiologically or induced by mechanical load. Researches on this subject report consistent opinions regarding biological resul ts. ${ }^{15,17,19,21,22,23,25,28,29}$ The onset of biological changes occurs through the action of stimuli triggered by root displacement in the PDL space, thereby establishing areas of tension and compression..$^{21}$ Roberts ${ }^{22}$ reported that maximum displacement of a rat first molar occurs in the PDL space after about three hours of movement induction. Subsequently, this stimulus triggers a series of events involving cellular differentiation and proliferation resulting in bone resorption and formation. . $^{8,15,17,25,28,29}$ 
Table 6 - Significance of intragroup comparison between means of different thicknesses of periodontal ligament in apical $(A)$, Medium (M) and cervical $(C)$ regions of mesiobuccal root $(\mathrm{Mb})$ of moved teeth $(\mathrm{m})$ in $\mathrm{Gl}$ or Gll or GIII.

\begin{tabular}{|c|c|c|c|c|c|}
\hline \multicolumn{2}{|c|}{ GI } & \multicolumn{2}{|c|}{ GII } & \multicolumn{2}{|c|}{ GIII } \\
\hline $\begin{array}{c}\text { Region / } \\
\text { Time }\end{array}$ & Mean & $\begin{array}{c}\text { Region / } \\
\text { Time }\end{array}$ & Mean & $\begin{array}{c}\text { Region / } \\
\text { Time }\end{array}$ & Mean \\
\hline AMb7m & $0.109(A)$ & AMb14m & $0.117(A)$ & AMb21m & $0.127(A)$ \\
\hline $\mathrm{MMb} 7 \mathrm{~m}$ & $0.148(B)$ & MMb14m & $0.139(B)$ & $\mathrm{MMb} 21 \mathrm{~m}$ & $0.130(A)$ \\
\hline $\mathrm{CMb} 7 \mathrm{~m}$ & 0.149 (B) & CMb14m & $0.140(B)$ & $\mathrm{CMb} 21 \mathrm{~m}$ & $0.131(\mathrm{~A})$ \\
\hline
\end{tabular}

*Different Letters: Statistically significant differences indicated by ANOVA, Kruskal-Wallis - $p<0.05$ and subsequent application of Tukey's test for difference identification.

Table 8 - Significance of intergroup comparison between means of different thicknesses of periodontal ligament in apical (A), Medium (M) and cervical $(C)$ regions of mesiobuccal root $(\mathrm{Mb})$ of moved teeth $(\mathrm{m})$ in $\mathrm{Gl}$ or Gll or GIII.

\begin{tabular}{cccccc}
\multicolumn{1}{c}{ A } & \multicolumn{2}{c}{ M } & \multicolumn{2}{c}{ C } \\
$\begin{array}{c}\text { Region / } \\
\text { Time }\end{array}$ & Mean & $\begin{array}{c}\text { Region / } \\
\text { Time }\end{array}$ & Mean & $\begin{array}{c}\text { Region / } \\
\text { Time }\end{array}$ & Mean \\
AMb7m & $0.109(\mathrm{~A})$ & MMb7m & $0.148(\mathrm{~A})$ & CMb7m & $0.149(\mathrm{~A})$ \\
AMb14m & $0.117(\mathrm{AB})$ & MMb14m & $0.139(\mathrm{AB})$ & CMb14m & $0.140(\mathrm{AB})$ \\
AMb21m & $0.127(\mathrm{~B})$ & MMb21m & $0.130(\mathrm{~B})$ & $\mathrm{CMb21m}$ & $0.131(\mathrm{~B})$ \\
\hline
\end{tabular}

* Different Letters: Statistically significant differences indicated by ANOVA, Kruskal-Wallis - $p<0.05$ and subsequent application of Tukey's test for difference identification.

The use of rats as an experimental framework over the years has enabled the solution of problems such as the lack of conclusive results involving clinical trials in humans. ${ }^{20}$ Murine molars exhibit limited development $^{5}$ so that the biological events that take place during OTM are very similar to those of humans but occur in a shorter period of time given these animals' accelerated metabolism. ${ }^{20}$

Although aware of this biological factor characterized by greater speed in the metabolic reactions of rats, ${ }^{20}$ which leads most researchers to use experimental times of 1, 3, 5 and 7 days, the authors of this study were interested in investigating the intensity of these reactions during the reactivation intervals applied to orthodontic appliances in humans, i.e., after at least 21 days following the application of force in order to add some new information to clinical practice.

Among the numerous limitations inherent in the histomorphometric technique, one should highlight the
Table 7 - Significance of intragroup comparison between means of different thicknesses of periodontal ligament in apical (A), Medium (M) and cervical $(C)$ regions of distobuccal root $(\mathrm{Mb})$ of moved teeth $(\mathrm{m})$ in $\mathrm{Gl}$ or Gll or GIII.

\begin{tabular}{|c|c|c|c|c|c|}
\hline \multicolumn{2}{|c|}{ GI } & \multicolumn{2}{|c|}{ GII } & \multicolumn{2}{|c|}{ GIII } \\
\hline $\begin{array}{c}\text { Region / } \\
\text { Time }\end{array}$ & Mean & $\begin{array}{c}\text { Region / } \\
\text { Time }\end{array}$ & Mean & $\begin{array}{c}\text { Region / } \\
\text { Time }\end{array}$ & Mean \\
\hline ADb7m & $0.148(A)$ & ADb14m & $0.139(A)$ & ADb21m & $0.130(A)$ \\
\hline MDb7m & $0.146(A)$ & MDb14m & $0.138(A)$ & MDb21m & $0.129(\mathrm{~A})$ \\
\hline CDb7m & $0.107(B)$ & CDb14m & $0.116(B)$ & CDb21m & $0.124(A)$ \\
\hline
\end{tabular}

*Different Letters: Statistically significant differences indicated by ANOVA, Kruskal-Wallis - $p<0.05$ and subsequent application of Tukey's test for difference identification.

Table 9 - Significance of intergroup comparison between means of different thicknesses of periodontal ligament in apical $(A)$, Medium (M) and cervical $(C)$ regions of mesiobuccal root $(\mathrm{Mb})$ of moved teeth $(\mathrm{m})$ in $\mathrm{Gl}$ or Gll or GIII.

\begin{tabular}{cccccc}
\multicolumn{1}{c}{ A } & \multicolumn{2}{c}{ M } & \multicolumn{2}{c}{ C } \\
$\begin{array}{c}\text { Region / } \\
\text { Time }\end{array}$ & Mean & $\begin{array}{c}\text { Region / } \\
\text { Time }\end{array}$ & Mean & $\begin{array}{c}\text { Region } \\
\text { / Time }\end{array}$ & Mean \\
ADb7m & $0.148(A)$ & MDb7m & $0.146(A)$ & CDb7m & $0.107(A)$ \\
ADb14m & $0.139(A B)$ & MDb14m & $0.138(A B)$ & CDb14m & $0.116(A B)$ \\
ADb21m & $0.130(B)$ & MDb21m & $0.129(B)$ & CDb21m & $0.124(B)$ \\
\hline
\end{tabular}

* Different Letters: Statistically significant differences indicated by ANOVA, Kruskal-Wallis - $p<0.05$ and subsequent application of Tukey's test for difference identification.

method of performing linear measurements on a large number of experimental models to obtain a mean value.

In this study, histometric analysis was performed in the longitudinal direction. To minimize the error potential described above, histological sections were obtained sequentially from 1 to 50 . Slides of histological sections numbers 24,25 and 26 , which were expected to display a larger mesiodistal size, were analyzed and the one with the best image quality was selected for subsequent histomorphometric analysis.

However, it should be noted that analysis of a histological section, be it quantitatively or qualitatively, is limited to the image being analyzed and often overlooks information concerning other regions.

As there was negligible or no inflammatory influence in the region, periodontal spaces were used in the inter-radicular septal region, which corresponds to the distal surface of the mesiobuccal root and the mesial surface of the distobuccal root. This event takes 
place in the mesial surface of the mesiobuccal root and distal surface of the distobuccal root and can lead to outcome interpretation errors.

The orthodontic force causes changes in the PDL, indicating that tooth movement has started. ${ }^{19}$ According to the literature, this force ranges between $10 \mathrm{~g}^{14,18}$ or even $30 \mathrm{~g}$ and 60 g. ${ }^{3,29,30} \mathrm{King}$ et $\mathrm{al}^{12}$ demonstrated no significant difference in the amount of OTM between $40 \mathrm{~g}$ and $60 \mathrm{~g}$, and further concluded that orthodontic appliances can be overloaded without increasing the amount of OTM. Brudvik and Rygh ${ }^{3}$ linked this result with the presence of hyaline areas that delay bone remodeling. Thus, a force of $40 \mathrm{cN}$ (equivalent to $40 \mathrm{~g}$ ) was employed in this experiment.

Isaacson et $\mathrm{al}^{11}$ pointed out that the unit of measure gram (g) is used to refer to mass and is therefore unsuitable to express force levels, which requires the use of the unit of measure Newton $(\mathrm{N})$. The conversion factors are: $1 \mathrm{~N}=101,937 \mathrm{~g}$ or $1 \mathrm{~g}=0.00981 \mathrm{~N}$. Whereas centi (c) is prepended to a unit of measure and forms the name of a derived unit 100 times smaller than the first, one can conclude that $1 \mathrm{~g}=0.981 \mathrm{cN}$, or that $1 \mathrm{~g}$ corresponds to approximately $1 \mathrm{cN}$.

Ashizawa and Sahara ${ }^{1}$ explained that in the early stages of OTM the magnitude of the initial force may not affect bone formation in the tension side but can influence the PDL condition on the compression side.

Measurements of PDL space in teeth undergoing OTM (GI, GII and GIII) were lower than in the control group and were construed as a state of PDL compression, while higher values indicated a PDL traction.

In this study, PDL width was approximately 0.13 $\mathrm{mm}$ in the apical, middle and cervical regions of murine molars not subjected to OTM, a value that proved similar to those observed in the literature. ${ }^{27}$

A comparison between GI linear values and those of the control group showed an occurrence of statistically significant difference in the three regions of the two roots (Fig 3). Apical regions of the mesiobuccal root and cervical regions of the distobuccal root exhibited lower values than the control group, and probably experienced the phenomenon of compression of PDL fibers. Moreover, the cervical regions of the mesiobuccal root, apical regions of the distobuccal root and middle regions of the both roots displayed higher values than control group, and may be related to changes in PDL traction direction. It should be noted that dimensional changes in OTM do not reflect the amount of tooth movement, highlighted by Baumrind ${ }^{2}$ as ten times greater than changes in PDL width.

These dimensional changes caused by OTM are related to a biological response after application of mechanical forces. This clearly demonstrated that the use of the method devised by Heller and Nanda ${ }^{9}$ induced tooth inclination, as previously described by Talic et al, ${ }^{26}$ since two completely distinct and opposed phenomena could be observed in the same root, be it mesiobuccal or distobuccal.

After 14 days of experiments, a statistically significant difference between GII and the control group continued to occur in the three regions of the two evaluated roots (Fig 4). The direction of the dimensional changes noted in GII remained the same as those observed in GI. However, change magnitude was lower in GII than in GI, i.e., the likelihood occurrence of PDL tension and compression sites was more pronounced at 7 days of OTM, a fact consistent with previous studies that explain the occurrence of tissue remodeling through alveolar bone resorption in the regions of compression and deposition of bone tissue in the portions where the PDL fibers were stretched, namely, in the tension areas. ${ }^{15,17,23,25,28,29}$

A comparison between GIII and control group values showed a lack of statistically significant difference in the three regions of the two evaluated roots (Fig 5). This equivalence between values shows a total reestablishment of the integrity of the PDL 21 days after the probable compression in the apical region of the mesiobuccal root and in the cervical region of the distobuccal root; and tension in the PDL fibers of the middle areas of both roots, cervical areas of mesiobuccal roots and apical areas of distobuccal roots. This decrease in the intensity to a level not statistically different from the control group corroborates the literature, indicating that the support periodontium is the site where tissue modifications caused by OTM take place, which results in the distribution and dissipation of mechanical stress., ${ }^{4,8}$

A comparison between the experimental groups whose teeth moved and the control group, whose teeth did not move in three different time periods, suggested the occurrence of alveolar bone remodeling and offered ideal conditions for observing morphological changes in PDL. ${ }^{14}$ 


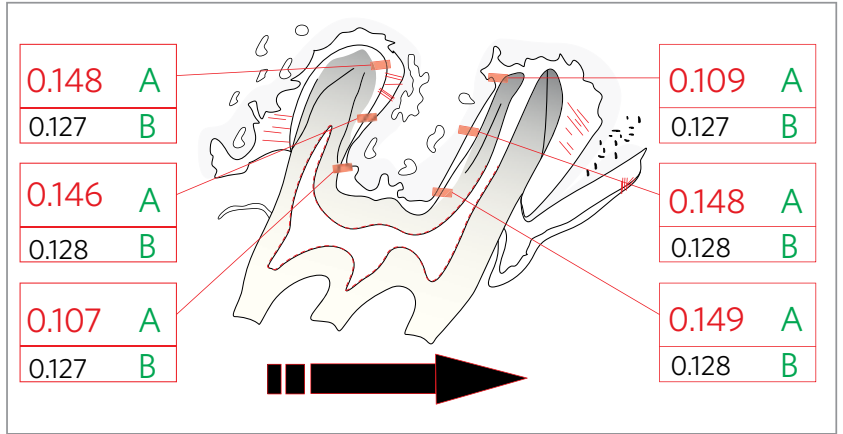

Figure 3 - Means of periodontal ligament dimensions in mesiobuccal and distobuccal roots in $\mathrm{Gl}$ and Control Group.

* Different Letters: Statistically significant differences $(p<0.05)$

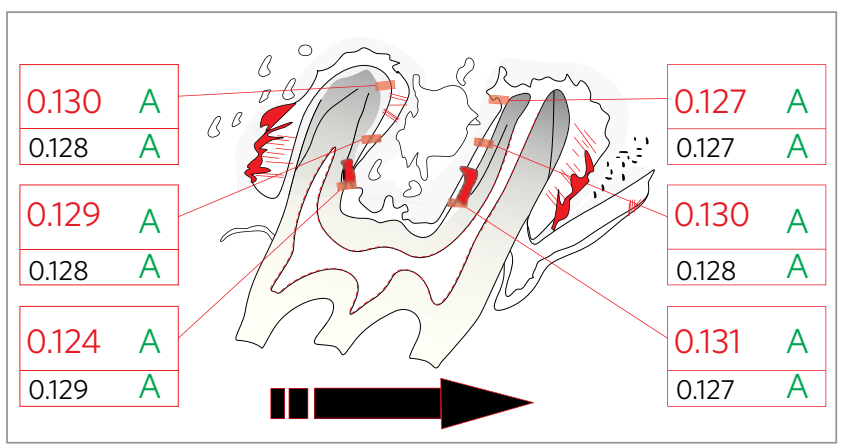

Figure 5 - Means of periodontal ligament dimensions in mesiobuccal and distobuccal roots in GIII and Control Group.

* Different Letters: Statistically significant differences $(p<0.05)$

The following comparative analyses considered only teeth that experienced movement. Initially, PDL size values were compared in the three different regions, i.e., apical, middle and cervical of the same root, mesiobuccal or distobuccal, individually in GI, GII or GIII.

The mesiobuccal root exhibited a significant PDL size difference in the apical region relative to the middle and cervical portions 7 days and 14 days into the experiment (Fig 6). Conversely, the middle and cervical regions exhibited no significant difference. Data from GI and GII showed that the apical region of the mesiobuccal root experienced a different phenomenon which occurred in the middle and cervical regions. ${ }^{26}$ Moreover, the magnitude of the changes in GI was higher than in GII. A comparison between the values obtained in the apical, middle and cervical regions in the mesiobuccal root of GIII showed statistical equivalence, suggesting that in the three regions

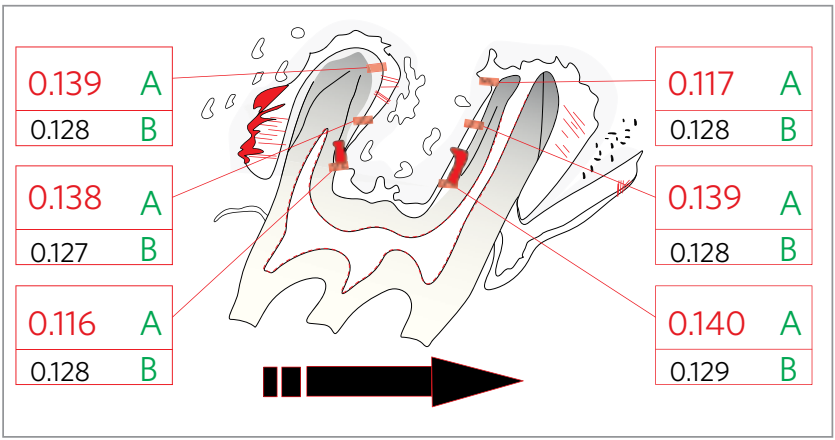

Figure 4 - Means of periodontal ligament dimensions in mesiobuccal and distobuccal roots in GIl and Control Group.

* Different Letters: Statistically significant differences $(p<0.05)$

(regardless of the phenomenon mentioned before) PDL restored its dimensions, indicating that bone remodeling occurred ${ }^{15,17,23,25,28,29}$ after 21 days of OTM.

A comparison between the values obtained in the three different regions of the distobuccal roots in GI and GII showed a significant difference in the cervical region relative to the middle and apical portions (Fig 7). In contrast, comparative analysis between the apical and middle regions showed no significant difference. Data from GI and GII showed that the cervical region of the distobuccal root experienced a different phenomenon which occurred in the middle and cervical regions ${ }^{26}$ and which allows one to assert that similarly to what had taken place in the mesiobuccal root the magnitude of the changes was higher in GI than in GII. A comparison between the three regions of the distobuccal roots in GIII showed no statistically significant differences. The PDL is the physiological mediator of orthodontic treatment, maintaining tooth positioning through the distribution of physiological and induced forces. ${ }^{1,15}$ In this sense, the histomorphometric data collected from GIII pointed to a likely restoration to normalcy in the three dimensional levels evaluated after 21 days into the experiment and confirmed PDL's role in the maintenance of periodontal homeostasis.

It is probable that the apical region of the mesiobuccal root and cervical region of the distobuccal root experienced PDL fiber compression. Moreover, the cervical regions of the mesiobuccal root, apical region of the distobuccal root and middle region of both roots may be related to PDL changes in the tension direction.

There was also concern in individually assessing changes in each region, be it the apical or middle or 


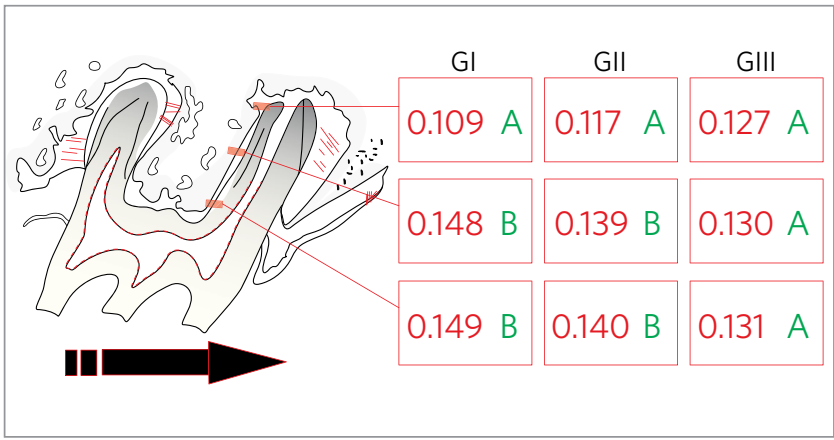

Figure 6 - Mean periodontal ligament dimensions in apical, middle and cervical regions of mesiobuccal root in Gl or GII or GIII.

${ }^{*}$ Different Letters: Statistically significant differences $(p<0.05)$.

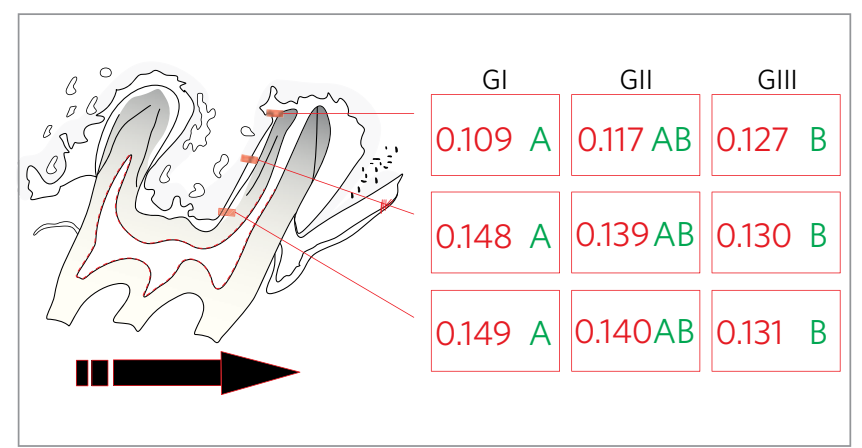

Figure 8 - Mean periodontal ligament dimensions in apical or middle or cervical regions of mesiobuccal root in $\mathrm{Gl}$, GII and GIII.

* Different Letters: Statistically significant differences $(p<0.05)$.

cervical in the mesiobuccal root (Fig 8) and distobuccal root (Fig 9) at three different times in groups GI, GII and GIII. These comparisons allowed equality between GI and GII values as well as between GII and GIII. The finding of a significant difference between GI and GII was noteworthy.

According to the literature, hyalinization in compression zones emerges at different times, depending on the intensity of the applied force. ${ }^{19}$ Its appearance has been reported to occur between three and six hours after force application, ${ }^{15}$ or else as a result of one day of tooth movement. ${ }^{3}$ The need to gradually remove this hyalinized ${ }^{23}$ tissue starting on the seventh day of experiment should be underlined. ${ }^{27}$ Cuoghi ${ }^{6}$ noted that in the first moments of OTM no change occurs in the microscopic morphology of the alveolar bone. OTM in the early stages is probably established at the expense of tooth displacement in the PDL and bone bending.

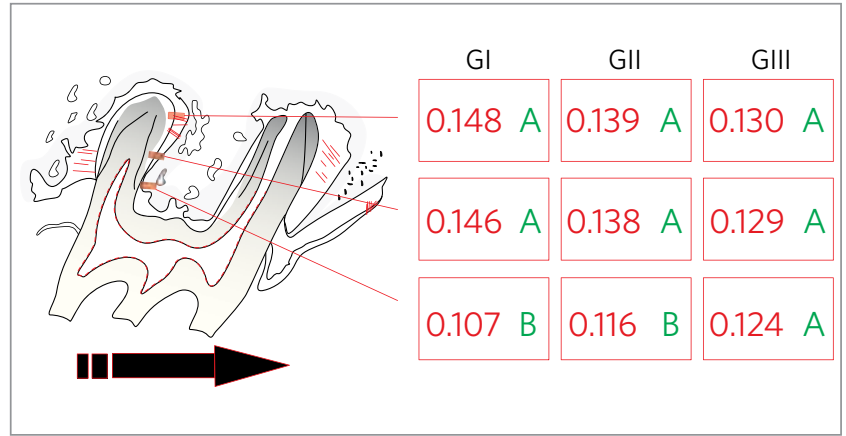

Figure 7 - Mean periodontal ligament dimensions in apical, middle and cervical regions of distobuccal root in GI or GII or GIII.

* Different Letters: Statistically significant differences $(p<0.05)$.

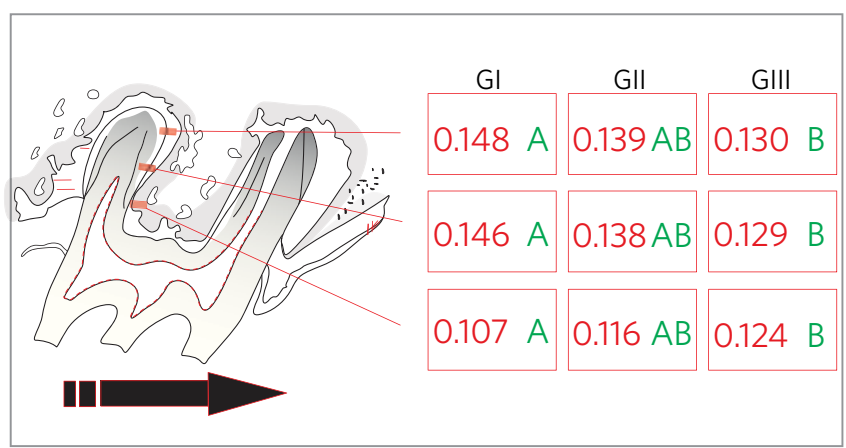

Figure 9 - Mean periodontal ligament dimensions in apical or middle or cervical regions of distobuccal root in GI, GIl and GIII.

* Different Letters: Statistically significant differences $(p<0.05)$.

Figures 8 and 9 suggest that potential hyaline areas present in the GI were probably experiencing a recovery process in GII and were virtually eliminated in GIII. Although in the same root PDL showed dimensional changes in different directions, the gradual return of linear values to homeostasis occurred in all areas examined in both roots, regardless of increases or decreases in PDL size. The hypothesis of hyalinized zones developing in areas of traction has not been ruled out.

Tooth movement consists of mechanical loading over teeth based on biomechanical principles. ${ }^{8}$ Investigation of biological phenomena is prompted by the desire to expand knowledge of these events in order to determine whether clinical orthodontics is effective and harmless. Furthermore, this goal stems from a perpetual quest to improve the clinical protocol, generating knowledge of principles and biological changes in tissues, cells and molecules. ${ }^{21}$ 
All OTM knowledge attained in the early days, ${ }^{8,16,19,21-24}$ in conjunction with information generated through the analysis of qualitative changes in the PDL ${ }^{15,17,19,21,23,25,28,29}$ and the results provided by morphometric research, all contribute to continued advancement in this line of investigation.

\section{CONCLUSIONS}

After 7 days of OTM, PDL dimensions were reduced in the apical region of the mesiobuccal root and cervical region of the distobuccal root, and were enlarged in the cervical region of the mesiobuccal root, apical region of the distobuccal root and middle region of both roots.
This reduction and enlargement in PDL size was observed in the same root, either mesiobuccal or distobuccal, and is representative of tooth inclination.

PDL size changes improved after 14 days of OTM and recovered normal control group values after 21 days.

Apical, middle and cervical regions were compared with each other in both roots and confirmed the occurrence of lingual inclination at 7 and 14 days of the experiment.

Individual assessment of each region (middle, apical or cervical) in the three time periods, confirmed that PDL dimensions were gradually restored in both roots, i.e., mesiobuccal and distobuccal.

\section{REFERENCES}

1. Ashizawa Y, Sahara N. Quantitative evaluation of newly formed bone in the alveolar wall surrounding the root during the initial stage of experimental tooth movement in the rat. Arch Oral Biol. 1998 Jun;43(6):473-84.

2. Baumrind S. A reconsideration of the propriety of the pressure-tension hypothesis. Am J Orthod. 1969 Jan;55(1):12-22.

3. Brudvik P, Rygh P. Non-clast cells start orthodontic root resorption in the periphery of hyalinized zones. Eur J Orthod. 1993 Dec;15(6):467-80.

4. Cho MI, Garant PR. Development and general structure of the periodontium Periodontol 2000. 2000 Oct;24:9-27.

5. Consolaro A, Martins-Ortiz MF. Um modelo experimental de movimentação dentária induzida e das reabsorções dentárias associadas. In: Consolaro A. Reabsorções dentárias nas especialidades clínicas. 2a ed. Maringá: Dental Press; 2005. p. 493-521.

6. Cuoghi OA. Avaliação macro e microscópica dos primeiros momentos da movimentação dentária induzida em macacos da espécie cebus apella [tese de doutorado]. Bauru (SP): Universidade de São Paulo, Faculdade de Odontologia de Bauru; 1996.

7. Dahlberg G. Statistical methods for medical and biological students. London: George Allen and Unwin; 1940.

8. Davidovicth Z. Tooth movement. Crit Rev Oral Biol Med. 1991;2(4):411-50

9. Heller IJ, Nanda R. Effect of metabolic alteration of periodontal fibers on orthodontic tooth movement. Am J Orthod. 1979 Mar;75(3):239-58.

10. Houston WJ. The analysis of errors in orthodontic measurements. Am J Orthod. 1983 May;83(5):382-90.

11. Isaacson RJ, Lindauer SJ, Davidovitch M. The ground rules for arch wire design. Semin Orthod. 1995 Mar;1(1):3-11.

12. King GJ, Keeling SD, McCoy EA, Ward TH. Measuring dental drift and orthodontic tooth movement in response to various initial forces in adult rats. Am J Orthod Dentofacial Orthop. 1991 May;99(5):456-65.

13. King GJ, Keeling SD, Wronski TJ. Histomorphologic and chemical study of alveolar bone turnover in response to orthodontic tipping. In: Carlson DS, Goldstein SA editors. Bone biodynamics in orthodontic and orthopedic treatment. Ann Arbor (MI): The University of Michigan; 1992. p. 281-97.

14. Kyomen S, Tanne K. Influences of aging changes in proliferative rat of PDL cells during experimental tooth movement in rats. Angle Orthod. 1997;67(1):67-72.

15. Macapanpan LC, Weinmann JP, Brodie AG. Early tissue changes following tooth movement in rats. Angle Orthod. 1954;24(2):79-95.

16. Oppenheim A. Tissue changes, particularly of the bone, incident to tooth movement. Trans Eur Orthod Soc. 1911;3:57-67.
17. Otero RL, Parodi RJ, Ubios AM, Carranza FA Jr, Cabrini RL. Histologic and histometric study of bone resorption after tooth movement in rats. J Periodontal Res. 1973;8(5):327-33.

18. Pavlin D, Dove SB, Zadro R, Gluhak-Heinrich J. Mechanical loading stimulates differentiation of periodontal osteoblasts in a mouse osteoinduction model: effect on type I collagen and alkaline phosphatase genes. Calcif Tissue Int. 2000 Aug;67(2):163-72

19. Reitan K. Continuous bodily tooth movement and its histological significance. Acta Odontol Scand. $1947 \mathrm{Fev} ; 7(2): 115-44$

20. Ren Y, Maltha JC, Kuijpers-Jagtman AM. The rat as a model for orthodontic tooth movement - a critical review and proposed solution. Eur J Orthod. 2004 Oct;26(5):483-90

21. Roberts WE. Fisiologia do osso, metabolismo e biomecânica na prática ortodôntica. In: Graber TM, Vanarsdall Júnior RL, editors. Ortodontia: princípios e técnicas atuais. 3a ed. Rio de Janeiro (RJ): Guanabara Koogan; 2002. p. 169-227.

22. Roberts WE, Garetto LP, Katona TR. Principles of orthodontic biomechanics: metabolic and mechanical control mechanisms. In: Carlson DS, Goldstein SA, editors Bone biodynamics in orthodontic and orthopedic treatment. Ann Arbor (MI): The University of Michigan; 1992. p. 189-255.

23. Rygh P. Ultrastructural changes in tension zones of rat molar periodontium incident to orthodontic tooth movement. Am J Orthod. $1976 \mathrm{Sep} ; 70(3): 269-81$.

24. Sandstedt $C$. Einige beitrage zur theorie der Zahn regulierung. Nord Tandl Tidssk 1904;4:236-56

25. Smith RK, Roberts WE. Cell kinetics of the initial response to orthodontically induced osteogenesis in rat molar periodontal ligament. Calcif Tissue Int. 1980;30(1):51-6.

26. Talic NF, Evans CA, Daniel JC, Zaki AE. Proliferation of epithelial rests of Malassez during experimental tooth movement Am J Orthod Dentofacial Orthop. 2003 May;123(5):527-33.

27. Tengku BS, Joseph BK, Harbrow D, Taverne AA, Symons AL. Effect of a static magnetic field on orthodontic tooth movement in the rat. Eur J Orthod. 2000 Oct;22(5):475-87.

28. Tran Van PT, Vignery A, Baron R. Cellular kinetics of the bone remodelling sequence in the rat. Anat Rec. 1982 Apr;202(4):445-51.

29. Tsay TP, Chen MH, Oyen OJ. Osteoclast activation and recruitment after application of orthodontic force. Am J Orthod Dentofacial Orthop. 1999 Mar;115(3):323-30.

30. Williams S. A histomorphometric study of orthodontically induced root resorption. Eur J Orthod. 1984 Feb;6(1):35-47. 